\title{
MRI in the Diagnosis and Monitoring of Multiple Sclerosis: An Update
}

\author{
M. P. Wattjes • M. D. Steenwijk $\cdot$ M. Stangel
}

Received: 31 March 2015 / Accepted: 26 June 2015 / Published online: 23 July 2015

(C) The Author(s) 2015. This article is published with open access at Springerlink.com

\begin{abstract}
Magnetic resonance imaging (MRI) is the most powerful tool for the early (differential) diagnosis of multiple sclerosis (MS) and has been part of the International Panel criteria $(2001,2005,2010)$ for more than 10 years. The role of brain and spinal cord MRI in the diagnosis of MS is well established. New MR techniques and markers will further improve the diagnostic value in a research and clinical routine setting. In addition to diagnosis, MRI is widely used for prognostic evaluation as well as treatment efficacy and safety monitoring. This field has gained importance with the introduction of new MS therapeutics. Therefore, the scope of MRI-guided MS disease monitoring has been widened to include rigorous treatment monitoring aiming at "no evidence of disease activity (NEDA)". Next, safety monitoring of treated MS patients has become major concern to enable early detection of opportunistic infections such as progressive multifocal leukoencephalopathy (PML). Driven by these new developments, recently published expert panel guidelines stressed the need for standardized imaging protocols and targeted specialized imaging markers for MS diagnosis and disease monitoring. This review article aims to give an update on the role of MRI in the diagnosis and monitoring of MS with particular
\end{abstract}

M. P. Wattjes $(\varangle) \cdot$ M. D. Steenwijk, MSc

MS Center and Department of Radiology \& Nuclear Medicine,

VU University Medical Centre,

De Boelelaan 1117,

1081 HV Amsterdam, Netherlands

e-mail: m.wattjes@vumc.nl

M. Stangel, MD

Clinical Neuroimmunology and Neurochemistry,

Department of Neurology, Hannover Medical School,

Carl-Neuberg-Str. 1,

30625 Hannover, Germany emphasis to treatment efficacy and safety, both in clinical practice and in research.

Keywords Multiple sclerosis - MRI · Disease monitoring $\cdot$ Safety monitoring $\cdot$ PML

\section{Introduction}

The importance of magnetic resonance imaging (MRI) in the diagnosis of multiple sclerosis (MS) has led to the incorporation of MRI criteria into the International Panel criteria for diagnosis (McDonald criteria) in 2001 and its role has become even more established with the subsequent 2005 and 2010 revisions [1]. Recent expert panel guideline papers have further stressed the relevance of MRI in the context of differential diagnosis. MRI is also an important tool to monitor MS disease activity. There is a need for standardized MRI protocols regarding image acquisition, timing, and frequency [2-4]. Conventional MRI parameters such as the number and distribution of focal T2 lesions and contrast-enhancing lesions are well established for diagnostic purposes. However, it has been conclusively shown that these MRI markers are rather unspecific for disease evolution characterization. In addition, conventional MRI measures show limited correlation with clinical outcome measures such as those measuring physical and cognitive decline. This is also referred to as the "clinico-radiological paradox of MS" (Fig. 1) [5]. Therefore, there is a crucial need for further imaging techniques focusing on more specific imaging markers in particular those related to neurodegenerative aspects of MS pathology.

The diagnostic role of MRI in MS has to a certain degree overshadowed the great potential of MRI for disease and treatment monitoring of MS patients. However, in recent 
Fig. 1 Three axial fluid attenuated inversion recovery (FLAIR) images illustrating the clinicoradiological paradox of multiple sclerosis: a 52-year-old male (benign) relapsing remitting multiple sclerosis (RRMS) patient, disease duration of 23 years, expanded disability status scale (EDSS) score of 2.5; b 50-year-old female RRMS patient, disease duration of 11 years, EDSS score of 3.0, c 53-year-old female primary progressive multiple sclerosis (PPMS) patient, disease duration of 7 years, EDSS of 4.5
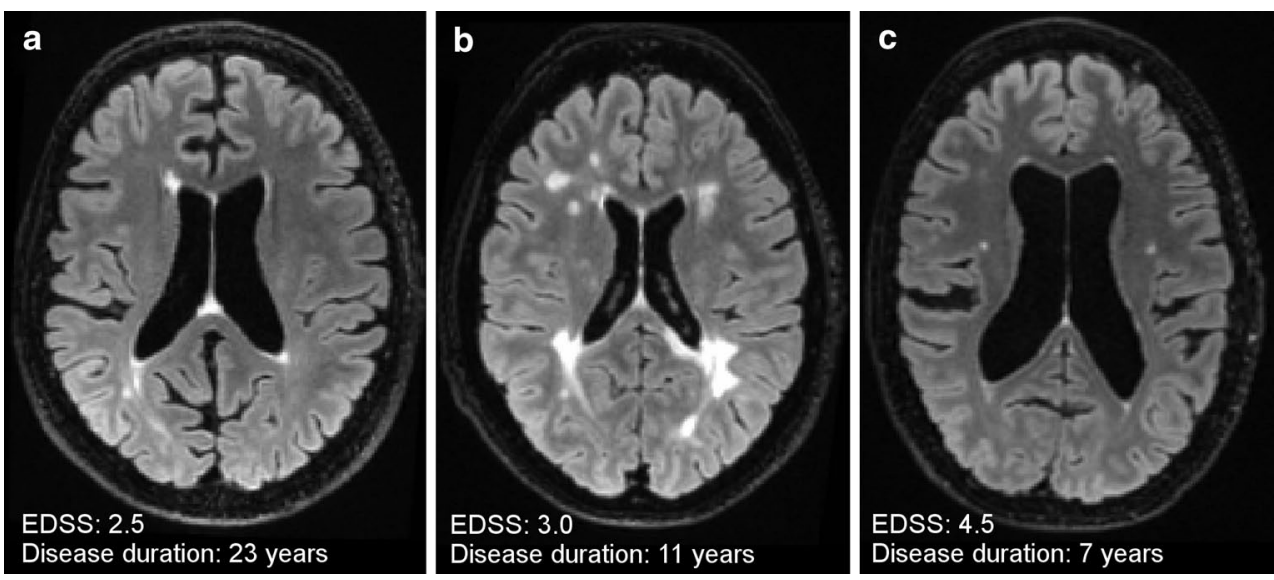

years, there has been substantial advancement in knowledge in this field of research, particularly with respect to alternative MRI methods and the concept of prediction of treatment efficacy and treatment safety monitoring. Therefore, the potential of MRI measures in assessing and monitoring treatment efficacy is increasingly being recognized and appreciated. With approval of the new and more effective generation of MS therapeutics, the spectrum of MRI in treatment monitoring has become broader, including the detection of opportunistic infections and paradoxical reactions (e.g., tumefactive demyelination). In addition, the emergence of immunomodulating drugs, which focus on alternative pharmacodynamic pathways for preventing MS disease progression (e.g., remyelination), require new imaging approaches to monitor disease activity [6].

The aim of this review is to give an update on advances in knowledge with respect to the role of MRI in the diagnosis and disease monitoring of MS in the context of new expert opinion guideline papers in this field.

\section{MRI in the Diagnosis of MS}

\section{Standardized MRI Protocol}

In the past decade, it has been conclusively demonstrated that changes in MRI acquisition parameters (e.g., magnetic field strength, pulse sequences, spatial resolution, coil technology, dose of contrast media) can substantially influence the detection of focal MS pathology. This has led to the widely accepted conclusion that standardized brain and spinal cord MRI protocols are required for diagnostic and monitoring purposes. However, even in the 2010 revisions of the International Panel (McDonald) criteria specific suggestions in terms of MRI acquisition are lacking. Recent expert panel guidelines have suggested a multisequence brain and spinal cord MRI protocol which is presented in Table 1 [2-4].
Brain MRI should be performed at a minimum magnetic field strength of $1.5 \mathrm{~T}(\mathrm{~T})$ while $3 \mathrm{~T}$ MRI shows increased sensitivity to focal MS lesion due to improved image resolution and signal-to-noise ratio, and therefore is recommended [7]. However, although it has been conclusively demonstrated that higher magnetic field strengths (e.g., $3 \mathrm{~T}$ ) do show improved sensitivity for white matter (WM) and grey matter (GM) lesions in clinically isolated syndrome (CIS) and MS patients as compared to standard field strengths $(1.5 \mathrm{~T})$, this does not have any consequences in terms of a possible earlier diagnosis of MS [8]. The spatial resolution of the 2D sequences should consist of a maximum slice thickness of $3 \mathrm{~mm}$ and an in-plane spatial resolution of $1 \times 1 \mathrm{~mm}$ (measured voxel size, $3 \times 1 \times 1 \mathrm{~mm}$ ). Spin echo or turbo (fast) spin echo proton-density (PD) and T2-weighted sequences are most frequently used and considered the reference standard due to their good sensitivity in detecting focal demyelinating lesions independent of their location. T2-weighted fluid attenuated inversion recovery (FLAIR) sequences show a higher sensitivity in detection of juxtacortical and periventricular lesions, but less sensitive in the posterior fossa. Isotropic 3D T2-weighted FLAIR is preferred due to the superior performance in terms of contrastto-noise ratio and the facilitation of isotropic multiplanar reconstructions, co-registration of longitudinal datasets, and application of fully automated lesion segmentation techniques [2].

In contrast to brain MRI, the acquisition of spinal cord images is more challenging due to the small tissue volume and the presence of artifacts caused by cerebrospinal fluid (CSF) flow and blood vessel pulsation. Conventional spin-echo sequences with cardiac gating may reduce vascular-related artifacts, but further increases the likelihood of movement artifacts due to an increased acquisition time [9]. Spinal cord MRI should be performed with MRI systems operating with a magnetic field strength of $1.5 \mathrm{~T}$ [2]. In contrast to brain MRI, there is no published evidence that $3 \mathrm{~T}$ image acquisition may lead to a higher sensitivity 
Table 1 Summary of a standardized MRI acquisition according to recent expert panel guidelines [2]

\section{Baseline MRI protocol}

Brain

Magnetic field strength: $1.5 \mathrm{~T}$ or $3 \mathrm{~T}$ (recommended)

Mandatory:

Axial 2D PD/T2-weighted spin echo or turbo (fast) spin echo Sagittal FLAIR (preferably 3D)

Axial post-contrast T1-weighted spin echo or turbo (fast) spin echo ${ }^{\mathrm{a}}$

Optional:

Double inversion recovery (preferably 3D) for cortical lesion detection

High-resolution isotropic 3D T1-weighted gradient echo for volumetric analysis

Diffusion and diffusion tensor imaging; Resting state fMRI

\section{Spinal cord}

Magnetic field strength: $1.5 \mathrm{~T}$ or $3 \mathrm{~T}$

Mandatory:

Sagittal 2D PD/T2-weighted spin echo or turbo (fast) spin-echo

Sagittal 2D contrast-enhanced T1-weighted spin echo ${ }^{\mathrm{a}}$

Optional:

Sagittal 2D STIR (as an alternative to proton-density-weighted)

Axial imaging: 2D T2 and/or contrast-enhanced T1-weighted ${ }^{\mathrm{a}}$

Follow-up MRI protocol

Brain

Magnetic field strength: $1.5 \mathrm{~T}$ or $3 \mathrm{~T}$ (recommended)

Mandatory sequences

Axial 2D PD/T2-weighted spin echo or turbo (fast) spin echo

Axial contrast-enhanced T1-weighted spin echo or turbo (fast) spin

echo

Optional sequences

Axial (2D or 3D) FLAIR as an alternative to PD,

Double inversion recovery (preferably 3D),

High-resolution isotropic 3D T1-weighted gradient echo

$\overline{T \text { Tesla, } D \text { dimensional, PDproton density, FLAIRfluid attenuated }}$ inversion recovery, STIRshort tau inversion recovery

${ }^{\text {a }}$ Single dose, $0.1 \mathrm{mmol} / \mathrm{kg}$ body weight

of lesion detection. Selection of an appropriate T2-weighted sequence is essential to obtain diagnostic valuable images. Sagittal image acquisition using 2D spin echo or turbo (fast) dual-echo (PD and T2-weighted) with a spatial resolution of at least $3 \times 1 \times 1 \mathrm{~mm}$ are considered to be the diagnostic standard (Table 1). Short-tau inversion recovery (STIR) T2-weighted sequences may be used as an alternative if PD-weighted images cannot be trusted as a consequence of flow-related artifacts possibly leading to false-positive results. T2-FLAIR lacks sensitivity for lesions in the spinal cord relative to conventional or fast $\mathrm{T} 2$-weighted sequences. Additional axial T2-weighted images can be obtained if a verification of changes identified in the sagittal plane is needed. The value of contrast administration in spinal cord MRI is still under discussion, since compared with brain lesions, only a small fraction of spinal cord lesions show contrast enhancement, and when it is seen, it is commonly associated with new clinical symptoms [9]. The updated imaging guidelines recommend a "one stop shop" strategy, in which spinal cord imaging is performed directly after contrast-enhanced brain imaging. This strategy saves time and reduces the need for additional contrast administration [2].

The Diagnostic Role of MRI According to MS Diagnostic Criteria

As a result of its high sensitivity to focal inflammatory demyelinating lesions, MRI has an important but also challenging role in especially the early MS disease course. This concerns in particular the establishment of an early (sensitive), but also a specific, diagnosis based on disease dissemination in space (DIS) and in time (DIT). MRI is able to detect MS disease activity with focal lesions in the brain and/or spinal cord while the patient may never have experienced any symptoms and therefore does not formally fulfill the McDonald criteria for MS (Fig. 2). This has led to the concept of radiologically isolated syndrome (RIS). RIS patients do show a higher risk for developing a CIS suggestive of MS (patient-reported or objectively observed events typical of an acute inflammatory demyelinating event in the CNS, current or historical, with duration of at least $24 \mathrm{~h}$ ) and later MS [1]. In order to correctly classify those patients with incidental brain lesions suggestive of MS pathology, recent diagnostic criteria for RIS have been proposed. These criteria include the number, location, and shape of the brain lesions [10].

In patients presenting with a CIS suggestive of MS, the 2010 revisions of the McDonald criteria allow for the first time the diagnosis of MS based on one single MRI scan showing DIS and DIT (a simultaneous presence of non-enhancing and non-symptomatic contrast-enhancing lesions). These criteria increased the sensitivity and simplified the features of both DIS and DIT, while maintaining the specificity of the earlier 2001 and 2005 McDonald criteria [1]. In addition to brain lesions, spinal cord lesions play an

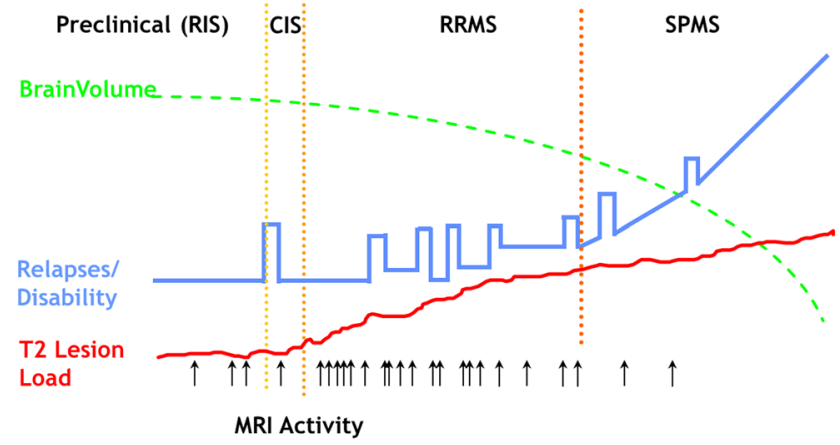

Fig. 2 Magnetic resonance imaging $(M R I)$ concept of multiple sclerosis in the context of disease course and clinical outcome measures. $R I S$ radiologically isolated syndrome $C I S$ clinically isolated syndrome, $R R M S$ relapsing remitting multiple sclerosis, SPMS secondary progressive multiple sclerosis 
important and powerful role in the diagnostic criteria, particularly for DIS [1, 10]. In those CIS patients not fulfilling 2010 McDonald diagnostic criteria, follow-up MRI scans are necessary to eventually establish the MS diagnosis. The interval between the baseline and follow-up scan is a matter of debate. Since $80 \%$ of the CIS patients with three or more brain lesions at baseline present with new T2 lesions after 3 months, a follow-up interval of 3-6 months has been recommended [2]. In the case of no DIT at that time, a third scan could be acquired 6-12 months later [11]. These time intervals may also apply to RIS patients. Although followup brain MRI is well established for demonstration of DIT and DIS, the value of repeated spinal cord imaging to establish the MS diagnosis is uncertain. Therefore serial spinal cord MRI not recommended in clinical routine for these purposes [12].

\section{New MRI Markers and Techniques for MS Diagnosis}

The 2010 revisions of the McDonald criteria also have limitations. For instance, imaging has gained substantial importance whereas other (para) clinical tests such as CSF analysis are rather neglected [13]. Another serious point of criticism is that the criteria may be too liberal possibly leading to a false positive MS diagnosis, especially in the presence of ischaemic small vessel disease (SVD). This has prompted the need for additional MRI approaches that help to differentiate lesions. The spinal cord is crucial in the differentiation of MS pathology from ischemic SVD [14]. In addition, the perivascular orientation of MS lesions (i.e., the observation that most MS lesions develop around a vessel) as a potential tool for lesion and disease differentiation has gained increasing scientific interest. By applying susceptibility-weighted imaging (SWI) techniques, it has been shown that the notion of a perivascular (perivenous) lesion orientation aids to differentiate MS lesions from focal vascular lesions (Fig. 3) or focal lesions of other MS mimics including neuromyelitis optica and Susac syndrome [15-17].

An additional point of criticism is the disregard of MS pathology beyond focal white matter lesions. Among these, GM pathology is of special interest, given its clinical relevance for cognitive dysfunction [18]. It has been shown that cortical GM lesions are rather specific for MS. The incorporation of cortical lesions into diagnostic criteria would further increase the specificity [19]. Additional pulse sequences such as the double inversions recovery (DIR) and the phase sensitive inversion recovery (PSIR) sequences have improved the detection rate of cortical GM lesions and the sensitivity can be even further increased by applying these pulse sequences at higher magnetic field strengths (Fig. 4) [20, 21]. However, it has been shown that the sensitivity of MRI using a dedicated protocol for grey matter
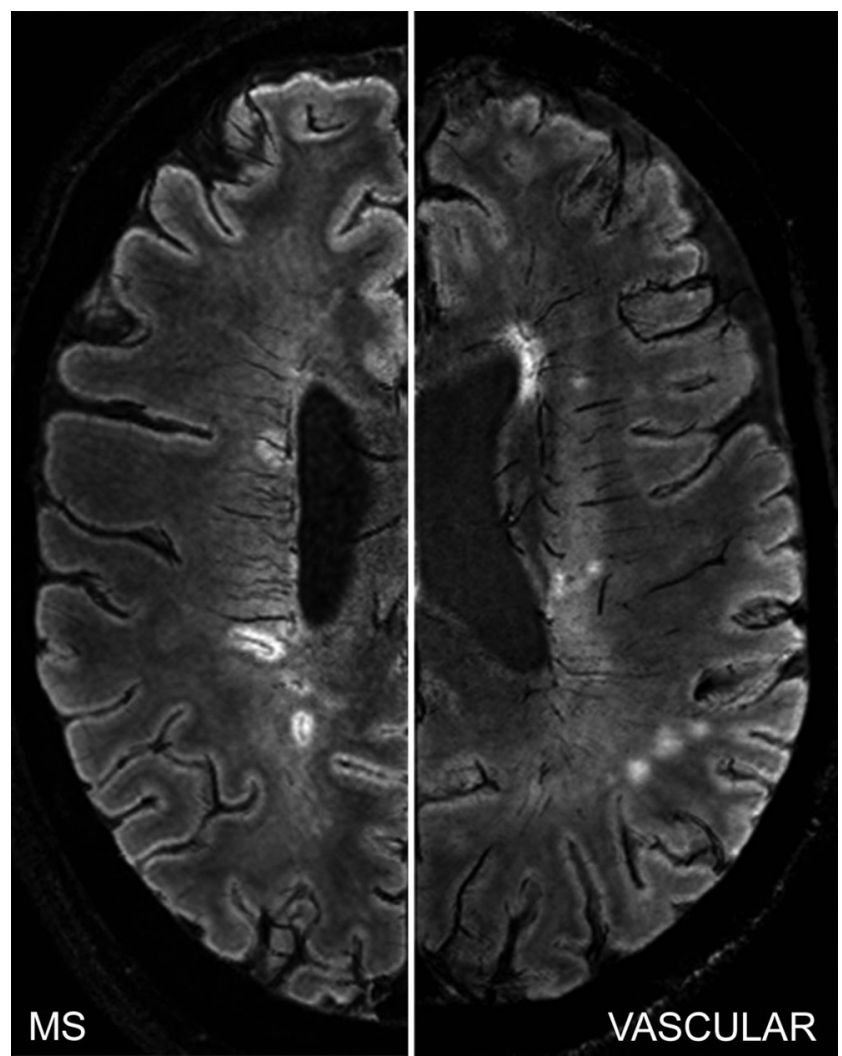

Fig. 3 Axial 7 T fluid attenuated inversion recovery (FLAIR)* images (combination of FLAIR and susceptibility-weighted imaging (SWI)) obtained in a multiple sclerosis $(M S)$ patient (left) and a patient with vasculo-ischemic small vessel disease (right). Please note that the MS lesion shows a central vein suggestive of perivenous inflammation whereas the vascular lesions do not show a central vein

lesion detection is far below the gold standard of histopathology [22]. In addition, there is a lack of standardization of image acquisition and image analysis of cortical lesions using dedicated imaging criteria on cortical GM lesions. These are important reasons why the presence of cortical lesions has not been incorporated into the McDonald criteria and is not used as an imaging marker for treatment trials on regular basis [23].

A promising tool for MS diagnosis is the next generation of high field MRI systems operating at $7 \mathrm{~T}$. However, whether in vivo $7 \mathrm{~T}$ MRI will improve the diagnostic accuracy and shed more light into the grey area of lesion heterogeneity needs to be investigated [24].

\section{MRI in MS Disease and Treatment Monitoring}

\section{MRI for Monitoring MS Disease Activity}

Several studies have demonstrated that a high lesion load and the location of MS lesions at the beginning of the disease is predictive for the development of clinical disabil- 
Fig. 4 Axial 3D fluid attenuated inversion recovery (FLAIR) images obtained at $7 \mathrm{~T}$ and $3 \mathrm{~T}$ showing a mixed grey matter-white matter lesion on the 7 T FLAIR image (arrow). This lesion was not prospectively identified at $3 \mathrm{~T}$

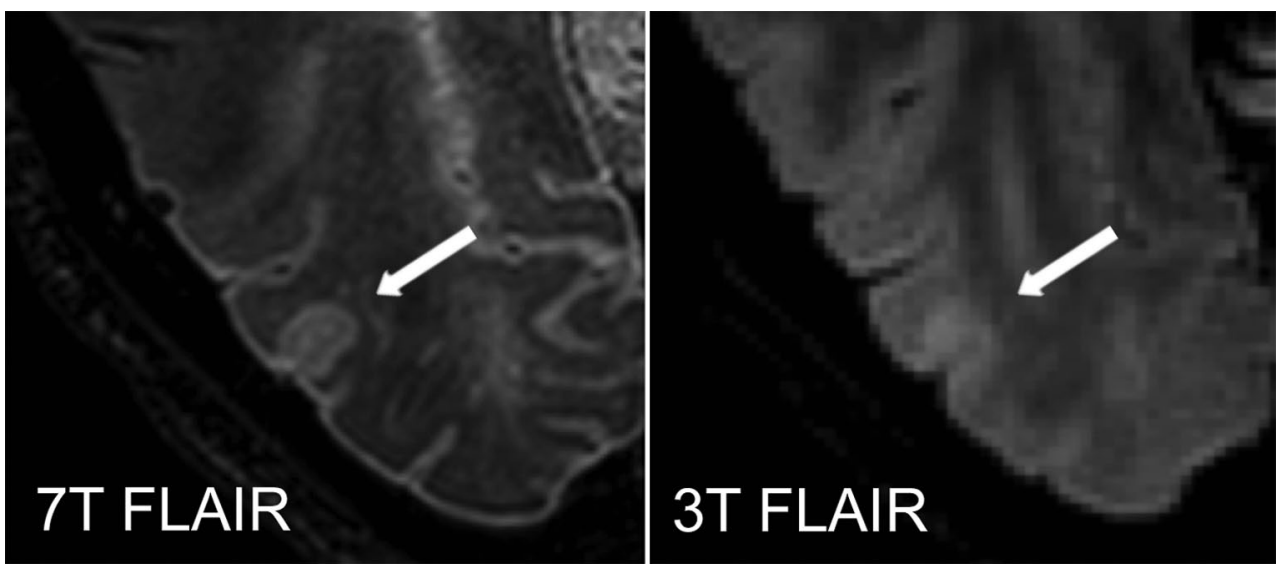

ity $[25,26]$. It is furthermore well established that disease activity as measured by MRI is more sensitive than the clinical disease activity as measured, for example, by the relapse rate. Thus, repeated MRI investigations are an established tool to detect and monitor subclinical disease activity. The most commonly used and recommended parameters that can be used in everyday practice are the detection of gadolinium (Gd)-enhancing $\mathrm{T} 1$ lesions and the detection of active (new or enlarging) T2 lesions [27, 28]. Although hypointense $\mathrm{T} 1$ lesions (black holes) correlate better with disability, this parameter reflects rather the persisting damage and not acute inflammatory disease activity.

Brain atrophy correlates best with clinical disease progression and acceleration of brain atrophy over time is thought to be a parameter of disease progression (Fig. 2) [29]. However, there are currently no standardized protocols to easily measure brain atrophy (see below). Thus new reliable and applicable methods to measure brain atrophy over time are required to include this parameter into routine investigations to monitor disease activity.

\section{MR Imaging in Monitoring and Predicting Treatment Response}

Almost all clinical trials investigating the clinical efficacy of a treatment in MS include standard MRI parameters (active T2 lesions, contrast-enhancing lesions) as secondary outcome measures. A recent meta-analysis of several treatment trials has demonstrated that an effect of the treatment on MRI lesions over a short period of 6-9 month in a phase 2 trial reliably predicts the effect on clinical relapse activity in a subsequent phase 3 trial with the same substance [30]. While the value of MRI measurements in treatment trials is acceptable, the utilization in everyday clinical practice has only been established in recent years. Several studies have demonstrated that the occurrence of new T2 lesions or Gd-enhancing T1 lesions during the first year of a treatment (most studies have investigated treatment with interferon- $\beta$ ) correlates with progression of disability [31, 32]. Thus, the detection of disease activity (or its absence) by MRI in a patient that receives an immunomodulatory treatment represents a measure of treatment response. This requires a baseline MRI before the initiation (or the change) of a treatment and a follow-up measurement. Due to pharmacodynamic differences, the time until MRI activity is suppressed differs between the immunomodulatory substances used in MS. For example, interferon- $\beta$ preparations reduce the number of Gd-enhancing reasons faster than glatiramer acetate [33]. Therefore some authors suggest that the reference scan should be obtained 3-6 month after treatment initiation/ change in order to overcome the uncertainty if the observed new lesions occurred before the treatment became effective. Further MRI scans should be obtained at intervals of 6-12 month in order to monitor subclinical MS activity [28].

\section{Advanced and New MRI Measures for MS Disease and Treatment Monitoring}

Given the modest correlation between established conventional MRI markers of MS disease activity (e.g., active T2 lesions, contrast-enhancing lesions) and clinical outcome measures, there is a crucial need for new and alternative MRI measures in the context of MS disease and treatment monitoring [4]. In particular, those measures focusing on aspects of MS disease pathology beyond focal inflammatory demyelinating lesions such as neurodegeneration and neuronal repair are of particular clinical relevance.

\section{Global Brain Volume Changes as a Marker of Neurodegeneration}

For a long time, neurodegeneration has been considered as an end-stage phenomenon of MS pathology. In fact, neurodegenerative changes such as volume loss in terms of brain atrophy becomes much more visible and evident in later disease stages. However, in the past few years it has been conclusively demonstrated that brain atrophy is present in the earliest stage of the MS disease, increases with disease 
duration, and may even accelerate in the progressive disease stages (Fig. 2). The clinical relevance of brain atrophy is well known: compared with measures of lesion load, brain atrophy shows better correlations with progression of disability and cognitive impairment, with GM atrophy being more closely associated with clinical dysfunction than WM atrophy [29].

In terms of GM involvement, it is known that both cortical and subcortical structures are affected. Of the subcortical structures, especially the thalamus is of interest, as thalamic atrophy is consistently reported present early in the disease and has a great relevance for cognitive dysfunction [34]. Reports on the spatial distribution of cortical GM atrophy are much sparse, but in the past years several studies consistently showed that especially the temporal lobe, precentral cortical areas, and medial parietal lobe are involved [35]. Although the pathophysiological background of brain atrophy in the context of MS pathology is complex and not completely understood, several hypotheses have been postulated [18]: they include primary damage of the GM [36], but also secondary damage due to axonal transection by lesions [37]. Multiple MRI studies consistently reported associations between whole-brain GM loss and increases of lesion load; however, recent reports suggest that the association between GM atrophy and WM pathology may be different depending on anatomical region and disease course $[38,39]$. In longstanding MS it was found that, whereas subcortical GM atrophy is associated with (connected) lesion load in both relapsing and progressive MS, cortical GM atrophy is more closely associated with normal appearing tissue integrity, but only in relapsing MS, and not in secondary progressive MS (Fig. 5).

Clearly, a better understanding and prevention of brain volume loss will have important clinical implications. Therefore, more recently, alternative treatment strategies beyond the control of inflammation focusing on neurodegenerative MS pathology have been developed. MRI brain volume measures have been repeatedly used in clinical trials to assess the effect of disease-modifying therapies. In a recent meta-analysis performed at the trial level, the overall effect of treatment on brain atrophy explained well the effect of treatment on disability [40]. Given the fact that the effect of a treatment on brain atrophy as measured by MRI correlates with clinical disability progression measured with the expanded disability status scale (EDSS), this has led to the proposal that these MRI measures should become an important outcome parameter in phase 2 and 3 trials. However, during the first 6 months-1 year of anti-inflammatory treatment, the brain volume may substantially decrease showing a subsequent stabilization during the second year of treatment. This phenomenon has been labeled as "pseudoatrophy", and it seems to be directly associated with resolution of ongoing white matter inflammation and edema induced by the anti-inflammatory drug at the time of treatment initiation $[29,41]$.

In addition to the whole brain volume assessment, the evaluation of those clinically relevant (e.g., cognition, fatigue) brain tissue substructures are becoming increasingly important. Among those, the volume of the thalamus, the medial temporal lobe, and the cortical grey matter thickness are the most relevant ones. However, the measurement of neurodegeneration is not limited to the brain tissue. The measurement of the mean upper cervical cord area (MUCCA) is a promising robust tool for these purposes [42]. Atrophy quantification in such substructures and the cervical cord has not been implemented in clinical trials yet; it would require the acquisition of additional 3D T1-weighted images.

\section{Measuring Remyelination and Neuronal Repair}

Neuroprotective drugs are increasingly becoming important in the field of MS research and treatment which is reflected by a broad spectrum of therapeutics currently being under

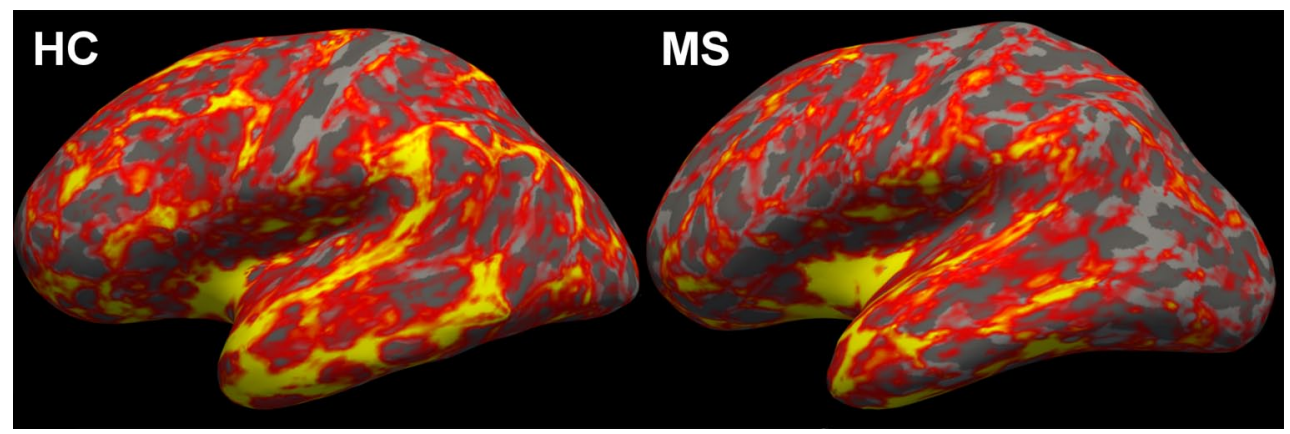

Fig. 5 Figure illustrating typical cortical grey matter atrophy in multiple sclerosis $(M S)$ : a 49 -year-old female healthy control $(H C)$, and a 50 -year-old female relapsing remitting multiple sclerosis (RRMS) patient (MS), disease duration of 11 years and expanded disability status scale (EDSS) score of 11 years. Both panels display an inflated cortical surface produced by FreeSurfer software, overlayed with vertex-wise cortical thickness (grey: $<2 \mathrm{~mm}$; red: $2 \mathrm{~mm}$; yellow: $>4 \mathrm{~mm}$ ) 
investigation in phase 1 and 2 clinical trials [43, 44]. Among these substances, remyelinating agents are of particular interest and clinical relevance in MS. Conventional MRI techniques are of limited value in monitoring remyelination. However, the measurement of the evolution of transient $\mathrm{T} 1$ hypointense lesions (black holes, $\mathrm{BH}$ ) as a potential marker is a well-established concept and is increasingly being applied in clinical treatment trials [45]. However, there is broad spectrum of advanced imaging techniques available allowing a more specific in vivo evaluation of the myelin content. This spectrum of methods includes magnetization transfer ratio (MTR), restricted proton fraction, diffusion tensor imaging (DTI), and positron emission tomography (PET) techniques. Among those, DTI and MTR are the most feasible techniques for clinical purposes. In particular, MTR can elegantly detect changes consistent with demyelination and remyelination. These changes can be observed within the same lesion, although they followed a different temporal evolution. Of note, signs of remyelination can be present even in lesions 3 years after their formation [46]. Although the uses of quantitative MRI measures for the measurement of remyelination or disease monitoring in general do have a promising potential, we have to be aware that their use in the clinical routine and multi-center setting is to a certain degree hampered by the longer acquisition, longer post-processing time and the lack of standardization.

\section{MRI for MS Pharmacovigilance}

Since the approval of the new and more effective generation of MS therapeutics the role of MRI in MS drug surveillance is increasingly gaining importance. The major aims of MRI drug surveillance includes the detection of unwished and unexpected MS disease activity (see above), paradoxical reactions (e.g., tumefactive demyelinating lesions), comorbidities (e.g., vascular, neoplastic), and unwanted side effects such as opportunistic infections [47].

The importance and clinical relevance of brain MRI for pharmacovigilance purposes has been well demonstrated in particular by studies monitoring MS patients treated with natalizumab (Biogen-Idec Inc., Cambridge, MA), a recombinant humanized monoclonal antibody against $\alpha 4$-integrin [48]. Progressive multifocal leukoencepalopathy (PML) is a relatively rare but serious side effect of natalizumab treatment. As of March 3rd 2015, 538 PML cases in natalizumab treated MS patients have been reported (Biogen Idec MedInfo. Available at: https://medinfo.biogenidec.com). It has been conclusively demonstrated that brain MRI is the most valuable screening method for the detection of PML being able to detect PML lesions in an early stage while the lesions are relatively small and the patients do not show any clinical symptoms suggestive of PML $[49,50]$. The clinical relevance of PML lesion detection in natalizumab-treated
MS patients at an asymptomatic stage is further stressed by positive effects on survival and functional outcome in the case of early and professional therapeutic intervention and patient management [51]. This has led to guidelines for MRI screening in natalizumab pharmacovigilance in terms of MRI protocols and frequency of MRI scanning. High risk patients (natalizumab treatment longer than 18 months, positive JC virus serostatus) should be screened every 3-6 months using at least an abbreviated MRI protocol including FLAIR, T2-weighted, and DWI sequences. Low risk patients (JC virus seronegative) should be monitored once a year [3]. Most of the knowledge on PML as an opportunistic infection has been derived from data of natalizumab treatment. However, we should be aware that MRI-based monitoring of patients for early PML detection is not exclusively recommended for those patients treated with natalizumab, but also for other MS drugs or the corresponding active substances, including alemtuzumab, rituximab, dimethylfumerate, and others [47, 52]. In addition, PML is not the only opportunistic infection which can be observed during MS treatment. MS therapeutics-related infections include a broad spectrum of pathogens including varizella zoster virus as has been shown in patients treated with fingolimod, a sphingosine 1-phosphate receptor modulator approved for MS treatment [53].

In addition, serious paradoxical reactions, such as tumefactive demyelination or overwhelming inflammatory demyelination, can occur during fingolimod treatment [54]. Given the growing number of emerging immunosuppressive and immunomodulating treatments for MS, MR-based safety monitoring will become increasingly more important and will be incorporated into future imaging guidelines for MS disease monitoring in the near future.

\section{Conclusion}

The role of MRI in the diagnosis and monitoring of MS is continuously gaining importance since we are increasingly able to detect and monitor MS pathology in a more sensitive and specific way. This leads to more individualized treatment strategies in MS patients including new generations of MS therapeutics targeting neuroprotection and remyelination. However, there is an ongoing need for additional conventional and quantitative MRI markers focusing on special and clinically relevant features of MS pathology. The standardization of these promising MRI methods for clinical routine and multicenter use will be a major challenge for the near future. In addition, there is a crucial need for the standardization of reading and interpretation of MS pathology on conventional MRI such as cortical grey matter lesions as well as for atrophy measurements. In addition, there is an unmet need for knowledge and guidance regarding the 
lesion interpretation on MR sequences with sensitivity specific pathology like iron (e.g., phase imaging). Implementation of the new generation of high-field MRI systems and their potential role in the detection and quantification of MS pathology has been investigated in relation with the MS diagnosis and differential diagnosis. Whether high-field MRI technology is also of use for MS disease monitoring must be further evaluated.

Acknowledgement The authors wish to thank Iris D. Kilsdonk for providing figure 3 and 4 .

Conflict of interest The authors report no conflict of interest regarding this work.

Open Access This article is distributed under the terms of the Creative Commons Attribution License which permits any use, distribution, and reproduction in any medium, provided the original author(s) and the source are credited.

\section{References}

1. Polman CH, Reingold SC, Banwell B, Clanet M, Cohen JA, Filippi M, Fujihara K, Havrdova E, Hutchinson M, Kappos L, Lublin FD, Montalban X, O'Connor P, Sandberg-Wollheim M, Thompson AJ, Waubant E, Weinshenker B, Wolinsky JS. Diagnostic criteria for multiple sclerosis: 2010 revisions to the McDonald criteria. Ann Neurol. 2011;69:292-302.

2. Rovira A, Wattjes MP, Tintoré M, Tur C, Yousry TA, Sormani MP, De Stefano N, Filippi M, Auger C, Rocca MA, Barkhof F, Fazekas F, Kappos L, Polman C, Miller D, Montalban X; MAGNIMS study group. Evidence-based guidelines: MAGNIMS consensus guidelines on the use of MRI in multiple sclerosis-clinical implementation in the diagnostic process. Nat Rev Neurol. 2015 Jul 7. [Epub ahead of print].

3. Wattjes MP, Rovira A, Miller DH, Yousry T, Sormani MP, de Stefano N, Tintoré M, Auger C, Tur C, Filippi M, Rocca MA, Fazekas F, Kappos L, Polman C, Barkhof F, Montalban X, on behalf of the MAGNIMS study group. MAGNIMS consensus guidelines on MR imaging in multiple sclerosis. MR imaging in multiple sclerosis disease prognosis and monitoring. Nat Rev Neurol. 2015 (submitted)

4. Petzold A, Wattjes MP, Costello F, Flores-Rivera J, Fraser CL, Fujihara K, Leavitt J, Marignier R, Paul F, Schippling S, Sindic C, Villoslada P, Weinshenker B, Plant GT. The investigation of acute optic neuritis: a review and proposed protocol. Nat Rev Neurol. 2014;10:447-58.

5. Barkhof F. The clinico-radiological paradox in multiple sclerosis revisited. Curr Opin Neurol. 2002;15:239-45.

6. Filippi M, Preziosa P, Rocca MA. Magnetic resonance outcome measures in multiple sclerosis trials: time to rethink? Curr Opin Neurol. 2014;27:290-9.

7. Wattjes MP, Lutterbey GG, Harzheim M, Gieseke J, Träber F, Klotz L, Klockgether T, Schild HH. Higher sensitivity in the detection of inflammatory brain lesions in patients with clinically isolated syndromes suggestive of multiple sclerosis using high field MRI: an intraindividual comparison of $1.5 \mathrm{~T}$ with $3.0 \mathrm{~T}$. Eur Radiol. 2006;16:2067-73.

8. Wattjes MP, Harzheim M, Lutterbey GG, Hojati F, Simon B, Schmidt S, Schild HH, Barkhof F. Does high field MRI allow an earlier diagnosis of multiple sclerosis? J Neurol. 2008;255:1159-63.
9. Gass A, Rocca MA, Agosta A, Ciccarelli O, Chard D, Valsasina P, Brooks JC, Bischof A, Eisele P, Kappos L, Barkhof F, Filippi M; MAGNIMS Study Group. MRI monitoring of pathological changes in the spinal cord in patients with multiple sclerosis. Lancet Neurol. 2015;14:443-54.

10. Okuda DT, Mowry EM, Beheshtian A, Waubant E, Baranzini SE, Goodin DS, Hauser SL, Pelletier D. Incidental MRI anomalies suggestive of multiple sclerosis: the radiologically isolated syndrome. Neurology. 2009;72(9):800-5. Erratum in: Neurology. 2009;72(14):1284.

11. Pestalozza IF, Pozzilli C, Di Legge S, Piattella MC, Pantano P, Caramia F, Pasqualetti P, Lenzi GL. Monthly brain magnetic resonance imaging scans in patients with clinically isolated syndrome. Mult Scler. 2005;11:390-4.

12. Sombekke MH, Wattjes MP, Balk LJ, Nielsen JM, Vrenken H, Uitdehaag BM, Polman CH, Barkhof F. Spinal cord lesions in patients with clinically isolated syndrome: a powerful tool in diagnosis and prognosis. Neurology. 2013;80:69-75.

13. Tumani H, Deisenhammer F, Giovannoni G, Gold R, Hartung HP, Hemmer B, Hohlfeld R, Otto M, Stangel M, Wildemann B, Zettl UK. Revised McDonald criteria: the persisting importance of cerebrospinal fluid analysis. Ann Neurol. 2011;70:520.

14. Bot JC, Barkhof $F$, Lycklama à Nijeholt $G$, van Schaardenburg D, Voskuyl AE, Ader HJ, Pijnenburg JA, Polman CH, Uitdehaag BM, Vermeulen EG, Castelijns JA. Differentiation of multiple sclerosis from other inflammatory disorders and cerebrovascular disease: value of spinal MR imaging. Radiology. 2002;223:46-56.

15. Kilsdonk ID, Wattjes MP, Lopez-Soriano A, Kuijer JP, de Jong MC, de Graaf WL, Conijn MM, Polman CH, Luijten PR, Geurts JJ, Geerlings MI, Barkhof F. Improved differentiation between MS and vascular brain lesions using FLAIR* at 7 T. Eur Radiol. 2014;24:841-9.

16. Wuerfel J, Sinnecker T, Ringelstein EB, Jarius S, Schwindt W, Niendorf T, Paul F, Kleffner I, Dörr J. Lesion morphology at 7 T MRI differentiates Susac syndrome from multiple sclerosis. Mult Scler. 2012;18:1592-9.

17. Sinnecker T, Dörr J, Pfueller CF, Harms L, Ruprecht K, Jarius S, Brück W, Niendorf T, Wuerfel J, Paul F. Distinct lesion morphology at 7-T MRI differentiates neuromyelitis optica from multiple sclerosis. Neurology. 2012;79:708-14.

18. Geurts JJ, Calabrese M, Fisher E, Rudick RA. Measurement and clinical effect of grey matter pathology in multiple sclerosis. Lancet Neurol. 2012;11:1082-92.

19. Filippi M, Rocca MA, Calabrese M, Sormani MP, Rinaldi F, Perini P, Comi G, Gallo P. Intracortical lesions: relevance for new MRI diagnostic criteria for multiple sclerosis. Neurology. 2010;75:1988-94.

20. Simon B, Schmidt S, Lukas C, Gieseke J, Träber F, Knol DL, Willinek WA, Geurts JJ, Schild HH, Barkhof F, Wattjes MP. Improved in vivo detection of cortical lesions in multiple sclerosis using double inversion recovery MR imaging at $3 \mathrm{~T}$. Eur Radiol. 2010;20:1675-83.

21. de Graaf WL, Kilsdonk ID, Lopez-Soriano A, Zwanenburg JJ, Visser F, Polman CH, Castelijns JA, Geurts JJ, Pouwels PJ, Luijten PR, Barkhof F, Wattjes MP. Clinical application of multicontrast 7-T MR imaging in multiple sclerosis: increased lesion detection compared to $3 \mathrm{~T}$ confined to grey matter. Eur Radiol. 2013;23:528-40.

22. Seewann A, Kooi EJ, Roosendaal SD, Pouwels PJ, Wattjes MP, van der Valk P, Barkhof F, Polman CH, Geurts JJ. Postmortem verification of MS cortical lesion detection with 3D DIR. Neurology. 2012;78:302-8. 
23. Geurts JJ, Roosendaal SD, Calabrese M, Ciccarelli O, Agosta F, Chard DT, Gass A, Huerga E, Moraal B, Pareto D, Rocca MA, Wattjes MP, Yousry TA, Uitdehaag BM, Barkhof F; MAGNIMS Study Group. Consensus recommendations for MS cortical lesion scoring using double inversion recovery MRI. Neurology. 2011;76:418-24.

24. Kilsdonk ID, de Graaf WL, Barkhof F, Wattjes MP. Inflammation high-field magnetic resonance imaging. Neuroimaging Clin $\mathrm{N}$ Am. 2012;22:135-57.

25. Fisniku LK, Brex PA, Altmann DR, Miszkiel KA, Benton CE, Lanyon R, Thompson AJ, Miller DH. Disability and T2 MRI lesions: a 20-year follow-up of patients with relapse onset of multiple sclerosis. Brain. 2008;131:808-17.

26. Tintore M, Rovira A, Arrambide G, Mitjana R, Río J, Auger C, Nos C, Edo MC, Castilló J, Horga A, Perez-Miralles F, Huerga E, Comabella M, Sastre-Garriga J, Montalban X. Brainstem lesions in clinically isolated syndromes. Neurology. 2010;75:1933-8.

27. Freedman MS, Selchen D, Arnold DL, Prat A, Banwell B, Yeung M, Morgenthau D, Lapierre Y; Canadian Multiple Sclerosis Working Group. Treatment optimization in MS: Canadian MS working group updated recommendations. Can J Neurol Sci. 2013;40:307-23.

28. Stangel M, Penner IK, Kallmann BA, Lukas C, Kieseier BC. Towards the implementation of 'no evidence of disease activity' in multiple sclerosis treatment: the multiple sclerosis decision model. Ther Adv Neurol Disord. 2015;8:3-13.

29. De Stefano N, Airas L, Grigoriadis N, Mattle HP, O'Riordan J, Oreja-Guevara C, Sellebjerg F, Stankoff B, Walczak A, Wiendl H, Kieseier BC. Clinical relevance of brain volume measures in multiple sclerosis. CNS Drugs. 2014;28:147-56.

30. Sormani MP, Bruzzi P. MRI lesions as a surrogate for relapses in multiple sclerosis: a meta-analysis of randomised trials. Lancet Neurol. 2013;12:669-76.

31. Prosperini L, Gallo V, Petsas N, Borriello G, Pozzilli C. One-year MRI scan predicts clinical response to interferon beta in multiple sclerosis. Eur J Neurol. 2009; 16:1202-9.

32. Río J, Castilló J, Rovira A, Tintoré M, Sastre-Garriga J, Horga A, Nos C, Comabella M, Aymerich X, Montalbán X. Measures in the first year of therapy predict the response to interferon beta in MS. Mult Scler. 2009;15:848-53.

33. Yong VW. Differential mechanisms of action of interferon-beta and glatiramer aetate in MS. Neurology. 2002;59:802-8.

34. Minagar A, Barnett MH, Benedict RH, Pelletier D, Pirko I, Sahraian MA, Frohman E, Zivadinov R. The thalamus and multiple sclerosis: modern views on pathologic, imaging, and clinical aspects. Neurology. 2013;80:210-9.

35. Steenwijk MD, Daams M, Pouwels PJ, J Balk L, Tewarie PK, Geurts JJ, Barkhof F, Vrenken H. Unraveling the relationship between regional grey matter atrophy and pathology in connected white matter tracts in long-standing multiple sclerosis. Hum Brain Mapp. 2015;36:1796-807.

36. Popescu V, Klaver R, Voorn P, Galis-de Graaf Y, Knol D, Twisk J, Versteeg A, Schenk G, Van der Valk P, Barkhof F, De Vries H, Vrenken H, Geurts J. What drives MRI-measured cortical atrophy in multiple sclerosis? Mult Scler. 2015 Jan 12. http://www.ncbi. nlm.nih.gov/pubmed/25583833. [Epub ahead of print].

37. Charil A, Dagher A, Lerch JP, Zijdenbos AP, Worsley KJ, Evans AC. Focal cortical atrophy in multiple sclerosis: relation to lesion load and disability. Neuroimage. 2007;34:509-17.

38. Steenwijk MD, Daams M, Pouwels PJ, Balk LJ, Tewarie PK, Killestein J, Uitdehaag BM, Geurts JJ, Barkhof F, Vrenken H. What explains grey matter atrophy in long-standing multiple sclerosis? Radiology. 2014;272:832-42.
39. Bergsland N, Laganà MM, Tavazzi E, Caffini M, Tortorella $\mathrm{P}, \mathrm{Ba}-$ glio F, Baselli G, Rovaris M. Corticospinal tract integrity is related to primary motor cortex thinning in relapsing-remitting multiple sclerosis. Mult Scler. 2015 Mar 19. http://www.ncbi.nlm.nih.gov/ pubmed/25791368. [Epub ahead of print].

40. Sormani MP, Arnold DL, De Stefano N. Treatment effect on brain atrophy correlates with treatment effect on disability in multiple sclerosis. Ann Neurol. 2014;75:43-9.

41. De Stefano N, Arnold DL. Towards a better understanding of pseudoatrophy in the brain of multiple sclerosis patients. Mult Scler 2015;21:675-6.

42. Daams M, Weiler F, Steenwijk MD, Hahn HK, Geurts JJ, Vrenken $\mathrm{H}$, van Schijndel RA, Balk LJ, Tewarie PK, Tillema JM, Killestein J, Uitdehaag BM, Barkhof F. Mean upper cervical cord area (MUCCA) measurement in long-standing multiple sclerosis: relation to brain findings and clinical disability. Mult Scler. 2014;20:1860-5.

43. Mallik S, Samson RS, Wheeler-Kingshott CA, Miller DH. Imaging outcomes for trials of remyelination in multiple sclerosis. $\mathrm{J}$ Neurol Neurosurg Psychiatry. 2014;85:1396-404.

44. Tran JQ, Rana J, Barkhof F, Melamed I, Gevorkyan H, Wattjes MP, de Jong R, Brosofsky K, Ray S, Xu L, Zhao J, Parr E, Cadavid $\mathrm{D}$. Randomized phase I trials of the safety/tolerability of antiLINGO-1 monoclonal antibody BIIB033. Neurol Neuroimmunol Neuroinflamm. 2014;1(2):e18.

45. Nagtegaal GJ, Pohl C, Wattjes MP, Hulst HE, Freedman MS, Hartung HP, Miller D, Montalban X, Kappos L, Edan G, Pleimes D, Beckman K, Stemper B, Polman CH, Sandbrink R, Barkhof F. Interferon beta- $1 \mathrm{~b}$ reduces black holes in a randomised trial of clinically isolated syndrome. Mult Scler. 2014;20:234-42.

46. Chen JT, Collins DL, Atkins HL, Freedman MS, Arnold DL. Magnetization transfer ratio evolution with demyelination and remyelination in multiple sclerosis lesions. Ann Neurol. 2008;63:254-62.

47. Rommer PS, Zettl UK, Kieseier B, Hartung HP, Menge T, Frohman E, Greenberg BM, Hemmer B, Stüve O. Requirement for safety monitoring for approved multiple sclerosis therapies: an overview. Clin Exp Immunol. 2014;175:397-407.

48. Rudick R, Polman C, Clifford D, Miller D, Steinman L. Natalizumab: bench to bedside and beyond. JAMA Neurol. 2013;70:172-82.

49. Wattjes MP, Richert ND, Killestein J, de Vos M, Sanchez E, Snaebjornsson P, Cadavid D, Barkhof F. The chameleon of neuroinflammation: magnetic resonance imaging characteristics of natalizumab-associated progressive multifocal leukoencephalopathy. Mult Scler. 2013;19:1826-40.

50. Wattjes MP, Vennegoor A, Steenwijk MD, de Vos M, Killestein J, van Oosten BW, Mostert J, Siepman DA, Moll W, van Golde AE, Frequin ST, Richert ND, Barkhof F. MRI pattern in asymptomatic natalizumab-associated PML. J Neurol Neurosurg Psychiatry. 2015;86:793-8.

51. Dong-Si T, Richman S, Wattjes MP, Wenten M, Gheuens S, Philip J, Datta S, McIninch J, Bozic C, Bloomgren G, Richert N. Outcome and survival of asymptomatic PML in natalizumab-treated MS patients. Ann Clin Transl Neurol. 2014;1:755-64.

52. van Oosten BW, Killestein J, Barkhof F, Polman CH, Wattjes MP. PML in a patient treated with dimethyl fumarate from a compounding pharmacy. N Engl J Med. 2013;368:1658-9.

53. Arvin AM, Wolinsky JS, Kappos L, Morris MI, Reder AT, Tornatore C, Gershon A, Gershon M, Levin MJ, Bezuidenhoudt M, Putzki N. Varicella-zoster virus infections in patients treated with fingolimod: risk assessment and consensus recommendations for management. JAMA Neurol. 2015;72:31-9.

54. Visser F, Wattjes MP, Pouwels PJ, Linssen WH, van Oosten BW. Tumefactive multiple sclerosis lesions under fingolimod treatment. Neurology. 2012;79:2000-3. 